\title{
On the Effect of Triplet or Doublet Chemotherapy in Advanced Gastric Cancer: Results From a National Cancer Registry
}

\author{
Alberto Carmona-Bayonas, MD, PhDa; Paula Jiménez-Fonseca, MD, PhDº ; Maria Luisa Sánchez Lorenzo, $\mathrm{MD}^{\mathrm{b}}$; \\ Avinash Ramchandani, MD; ; Elena Asensio Martínez, MDd; Ana Custodio, MD, PhD ; Marcelo Garrido, MD, PhD; \\ Isabel Echavarría, MD; ; Juana María Cano, $\mathrm{MD}^{\mathrm{h}}$; Jose Enrique Lorenzo Barreto, MD; Teresa García García, MD, PhD; \\ Felipe Álvarez Manceñido, MD; Alejandra Lacalle, MD; Marta Ferrer Cardona, MD'; Monserrat Mangas, MDm;

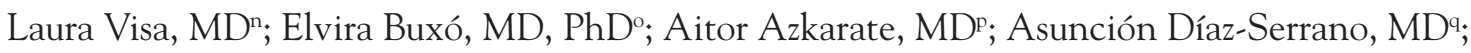 \\ Ana Fernández Montes, $\mathrm{MD}^{\mathrm{r}}$; and Fernando Rivera, $\mathrm{MD}, \mathrm{PhD}^{\mathrm{s}}$
}

\begin{abstract}
Background: There is currently no consensus regarding first-line chemotherapy for patients with advanced gastric cancer (AGC) who are ineligible to receive trastuzumab. The objective of this study was to evaluate the efficacy and tolerance of triplets versus doublets by analyzing a national gastric cancer registry. Patients and Method: Patients with AGC treated with polychemotherapy without associating trastuzumab were included from 2008 through 2016. The effect of triplets versus doublets was compared using 3 methods: Cox proportional hazards regression, propensity score matching (PSM), and coarsened exact matching (CEM). Results: $A$ total of 970 patients were recruited (doublets: $n=569 ;$ triplets: $n=401$ ). In the multivariate Cox model, the use of triplets was associated with better overall survival (OS), with a hazard ratio (HR) of $0.84(95 \% \mathrm{Cl}, 0.72-0.98$; $P=.035)$. After PSM, the sample contained 340 pairs. A significant increase in OS, 11.14 months ( $95 \% \mathrm{Cl}, 9.60-12.68)$ versus 9.60 months $(95 \% \mathrm{Cl}$, 8.44-10.75), was seen in favor of triplets ( $\mathrm{HR}, 0.77 ; 95 \% \mathrm{Cl}, 0.65-0.92$; stratified log-rank test, $P=.004$ ). The effect appeared to be comparable for anthracycline-based (HR, 0.78; 95\% Cl, 0.64-0.94) or docetaxel-based triplets $(\mathrm{HR}, 0.78 ; 95 \% \mathrm{Cl}, 0.60-1.009)$. The trend was similar after applying the CEM algorithm, with an HR of $0.78(95 \% \mathrm{Cl}, 0.63-0.97 ; \mathrm{P}=.03)$. Triplet therapy was viable and relative dose intensities exceeded $85 \%$, except for cisplatin in DCX (docetaxel, cisplatin, capecitabine). Triplets had more severe toxicity overall, especially hematologic, hepatic, and mucosal adverse events. Conclusions: With the limitations of a retrospective study that examines a heterogeneous set of chemotherapy regimens, we found that triplets are feasible in daily practice and are associated with a discreet benefit in efficacy at the expense of a moderate increase in toxicity.
\end{abstract}

J Natl Compr Canc Netw 2016;14(11):1379-1388

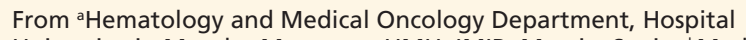
Universitario Morales Meseguer, UMU, IMIB, Murcia, Spain; ${ }^{b}$ Medical Oncology Department, Hospital Universitario Central de Asturias, Oviedo, Spain; 'Medical Oncology Department, Hospital Universitario Insular de Gran Canaria, Las Palmas, Spain; ${ }^{d}$ Medical Oncology Department, Hospital General Universitario de Elche, Alicante, Spain; 'Medical Oncology Department, Hospital Universitario La Paz, Madrid, Spain; ${ }^{f}$ Hematology and Medical Oncology Department, Hospital Universitario de Ciudad Real, Cuidad Real, Spain; ${ }^{9}$ Medical Oncology Department, Hospital Universitario Gregorio Marañón, Madrid, Spain; 'Medical Oncology Department, Hospital General de Ciudad Real, Ciudad Real, Spain; 'Medical Oncology Department, Hospital Universitario de Canarias, Santa Cruz de Tenerife, Spain; 'Pharmacy Department, Hospital Universitario Central de Asturias, Oviedo, Spain; ${ }^{k}$ Medical Oncology Department, Complejo Hospitalario de Navarra, Pamplona, Spain; 'Medical Oncology Department, Hospital Universitario Parc Tauli, Sabadell, Spain; ' Medical Oncology Department, Hospital Galdakao-Usansolo, Usansolo, Spain; "Medical Oncology Department, Hospital Universitario del Mar, Barcelona, Spain; -Medical Oncology Department, Hospital Clínic, Barcelona, Spain; ${ }^{\text {PMedical }}$ Oncology Department, Hospital Universitario Son Espases, Mallorca, Spain; "Medical Oncology Department, Hospital Universitario Doce de Octubre, Madrid, Spain; 'Medical Oncology Department, Complejo Hospitalario de
Orense, Spain; and sMedical Oncology Department, Hospital Universitario de Valdecilla, Santander, Spain.

Submitted June 11, 2016; accepted for publication July 21, 2016.

Dr. Carmona-Bayonas has disclosed receiving grant support from Ipsen; and serving in a consulting/advisory role for Pfizer. Dr. Jiménez-Fonseca has disclosed serving in a consulting/advisory role for Pfizer. Dr. Custodio has disclosed serving in a consulting/advisory role for Novartis, Pfizer, and Ipsen; and serving on a speakers' bureau for Novartis, Pfizer, and Ipsen. Dr. Rivera has disclosed receiving honoraria for certified continuing education from Roche, sanofi-aventis, Bayer, Merck/Serono, Amgen, Lilly, and Celgene; serving in a consulting/advisory role for Roche, sanofi-aventis, Bayer, Merck/ Serono, Amgen, Lilly, and Celgene; and receiving research support from Roche, sanofi-aventis, Bayer, Merck/Serono, Amgen, Lilly, and Celgene. The remaining authors have disclosed that they have no financial interests, arrangements, affiliations, or commercial interests with the manufacturers of any products discussed in this article or their competitors.

This is an academic study; the registry was supported by the authors. Correspondence: Alberto Carmona-Bayonas, MD, PhD, Hematology and Medical Oncology Department, Hospital Universitario Morales Meseguer, Calle Marqués de los Vélez s/n, 30008, Murcia, UMU, IMIB, Murcia, Spain.

E-mail: alberto.carmonabayonas@gmail.com 


\section{Background}

Advanced gastric cancer (AGC) continues to be one of the most common cancers in the world and a leading cause of cancer death. ${ }^{1}$ In patients with AGC that amplify or overexpress HER2, the standard of care combines cisplatin and fluoropyrimidines with trastuzumab. ${ }^{2}$ However, depending on location and histologic subtype, these tumors account for a mere $9 \%$ to $36 \%$ of all gastroesophageal cancers. ${ }^{3,4}$ For the remaining cases, there is no clear consensus as to the optimal scheme, with response rates in the range of 35\% to $45 \%$ for most regimens, and median overall survival (OS) that rarely exceeds 12 months for HER2-negative tumors.

The selection of doublet or triplet schedules is an unresolved issue that arises frequently in planning treatment for patients with AGC. ${ }^{5}$ In the V325 randomized clinical trial (RCT), the DCF (docetaxel, cisplatin, fluorouracil) regimen was superior to $\mathrm{CF}$ (cisplatin, fluorouracil) in patients with a good performance status (PS) and preserved organ function, although the extra benefit was scant and entailed elevated toxicity. ${ }^{6}$ One of the questions these data pose is to what degree they can be generalized to daily clinical practice, where extrapolation is complicated, given the chronic comorbidities, advanced age, or functional situation present in many of the patients. In fact, several groups have attempted to develop modified docetaxel-based triplets with improved tolerance profiles that better suit daily practice. ${ }^{7-10}$ However, with the exception of one study that looked at reduced-dose triplet therapy (miniDOX) specifically in a suboptimal population, ${ }^{10}$ the remaining trials included selected fit patients.

With respect to anthracyclines, a meta-analysis has suggested a significant benefit in OS in favor of epirubicin-containing schedules (hazard ratio [HR], 0.77; 95\% CI, 0.62-0.95). ${ }^{11}$ However, the study providing the largest weight in this meta-analysis was a comparison between 2 triplets with or without epirubicin, ${ }^{12}$ whereas the remaining 2 trials were limited by their small sample sizes. ${ }^{13,14}$ More recently, a second meta-analysis that specifically sought to assess the effect of triplets versus doublets showed better OS (HR, 0.90; 95\% CI, 0.83-0.97) and progressionfree survival (PFS) (HR, 0.80; 95\% CI, 0.69-0.93) in favor of triplets. ${ }^{15}$ Nevertheless, the addition of an anthracycline to a doublet did not attain statistical significance for OS; consequently, the beneficial effect of these agents on OS remains doubtful.
For all these reasons, the selection of triplets versus doublets is actually one of the most important uncertainties hindering the decision-making process in patients with AGC. We report an analysis of a national registry of AGC in an attempt to determine the efficacy and tolerance of triplet versus doublet regimes in daily clinical practice.

\section{Patients and Methods}

\section{Study Population}

The patients included in this analysis come from a national registry of AGC belonging to 28 centers. This registry comprises adult patients (aged $\geq 18$ years) with pathologically confirmed, unresectable, or metastatic gastric, gastroesophageal junction, or distal esophageal adenocarcinoma, who received at least one cycle of polychemotherapy. Exclusion criteria involved patients whose chemotherapy regimen was not coded, those for whom follow-up was less than 3 months (except for individuals with early death during this period, who have been included), those for whom it was less than 6 months since completion of prior neoadjuvant or adjuvant treatment, and those with other synchronous cancers. Patients who received trastuzumab in first-line therapy were excluded. All the patients were treated between 2008 and 2016 .

\section{Variables}

The covariates potentially involved in choosing chemotherapy doublets and triplets were selected ex ante: patient characteristics such as age, ECOG PS scale, and chronic comorbidities; tumor-dependent characteristics such as stage, tumor site, number of metastatic sites, baseline carcinoembryonic antigen (CEA) value, surgery of primary tumor, and the presence of serious cancer-derived complications at diagnosis (liver, respiratory, or renal dysfunction; intestinal obstruction; massive ascites; major bleeding; thromboembolic disease); and histologic features such as HER2 overexpression, Lauren classification, presence of signet ring cells, and histologic grade. The primary tumor site was coded as "esophageal" for Siewert I and II distal esophageal tumors; the remaining tumor locations were coded as "gastric cancer." Dose intensity was defined as the drug dose delivered per time unit and was expressed as $\mathrm{mg} / \mathrm{m}^{2}$ per week. Cumulative dose was defined as total dose and expressed as $\mathrm{mg} / \mathrm{m}^{2}$. Relative dose intensity (RDI) was defined as the dose intensity delivered rel- 
ative to the planned dose-intensity. Tumor response assessment was performed by the local researchers, who re-evaluated the CT images obtained as per the standard practice at each site approximately every 3 months, following RECIST 1.1 criteria.

\section{Statistical Considerations}

We applied 3 methods to evaluate the effect of triplets and doublets. First, we fitted a Cox proportional hazards regression model for OS including therapy and all the aforementioned covariates. Second, we applied propensity score estimation and matching. Third, coarsened exact matching (CEM) was used in an attempt to improve the estimation of causal effects.

After fitting a binary logistic regression model, 9 variables were chosen as the basis for propensity score matching (PSM). ${ }^{16}$ One-to-one matching without replacement was used to match patients who received doublet versus triplet chemotherapy. The matched samples were obtained by nearest neighbor matching with a caliper width of 0.2 . The standardized differences method was applied to assess the balance diagnostics. ${ }^{16,17}$ In general, standardized differences less than $10 \%$ indicate a proper balance between baseline variables. ${ }^{18}$

CEM is a monotonic imbalance bounding matching method in which adjusting the imbalance on one variable has no effect on the maximum imbalance of any other. ${ }^{19}$ We used the same variables mentioned earlier. To improve the matching procedure, the covariate "stage at diagnosis" was substituted for "prior perioperative treatment." The final result affords added advantages, such as less model dependence and possibly less bias. ${ }^{20}$

After applying the PSM and CEM algorithms, Kaplan-Meier survival curves were estimated separately for patients treated with triplets or doublets in the matched samples. Participants were stratified into 5 approximately equal-size subsets using the quintiles of the estimated propensity score. Stratified log-rank tests were applied after matching to compare the survival functions of triplets and doublets. ${ }^{17} \mathrm{CEM}$ requires weighting observations to compensate for the differential strata size. ${ }^{21,22}$ We estimated a sample size requirement of 325 matched pairs for a 1-year survival rate of $40 \%$ for triplets versus $30 \%$ for doublets, assuming a 2 -sided $5 \%$ significance level and $80 \%$ power.

STATA 14 software (StataCorp, College Station, TX) was used throughout.

\section{Results}

\section{Patients}

At the time of analysis (April 2016), the registry contained 1,615 patients, of whom 970 met the selection criteria for this analysis. Figure 1 provides a flowchart of patients in the registry. At this time point, $79.6 \%$ of the patients were dead, with a median OS of 8.38 months (95\% CI, 7.89-9.04). Firstline chemotherapy schemes are shown in supplemental eTables 1 and 2 (available with this article at JNCCN.org). Triplets were administered in $41.3 \%$ of patients $(n=401)$ and doublets in $58.7 \%(n=565)$. In the binary logistic regression (eTable 3), the variables associated with the use of triplets were (1) not having a chronic cardiopathy; (2) good PS (ECOG PS 0-1); (3) age; (4) unresectable, locally advanced tumors (vs metastatic disease); (5) extrahepatic metastases; (6) diffuse tumors; (7) poorly differentiated (G3) tumors; and (8) no prior perioperative chemotherapy.

\section{Population Characteristics Before and After PSM}

The study population consisted of 680 cases after PSM (340 patients who received doublets and 340 who were administered a triplet chemotherapy scheme). In eFigures 1 and 2 , the distribution of propensity scores and absolute standardized differences are presented before and after matching. Table 1 lists patient characteristics before and after PSM. PSM is effective in reducing absolute standardized differences for all categories. Impor-

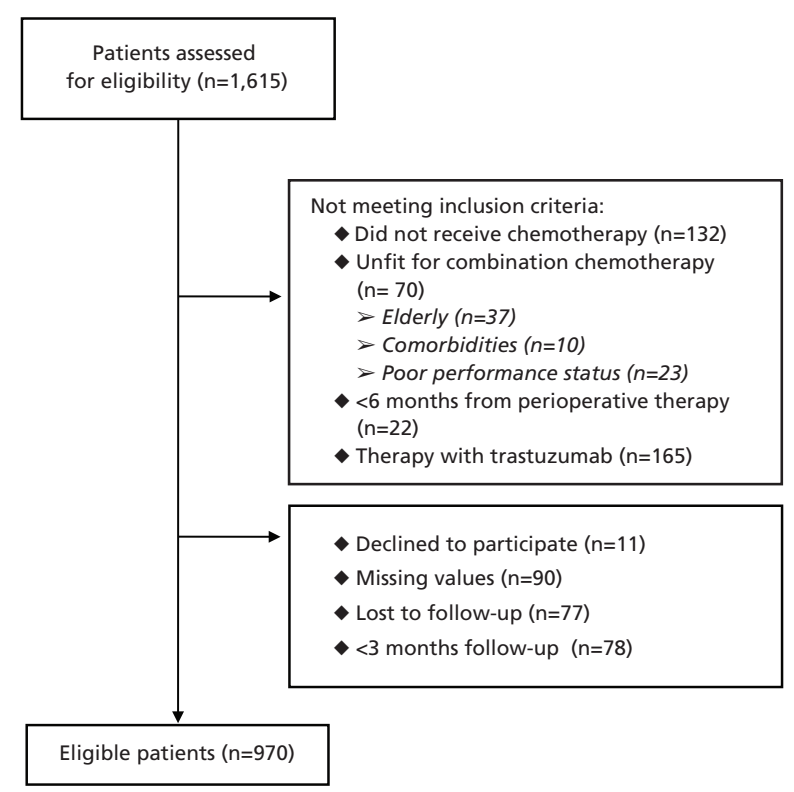

Figure 1. Flowchart of patients in the registry. 
Carmona-Bayonas et al

Table 1. Baseline Characteristics of Patients Treated With Triplets and Doublets

\begin{tabular}{|c|c|c|c|c|c|c|}
\hline \multirow[b]{2}{*}{ Characteristics } & \multicolumn{3}{|c|}{ Before PSM } & \multicolumn{3}{|c|}{ After PSM } \\
\hline & $\begin{array}{l}\text { Doublet } \\
(n=569)\end{array}$ & $\begin{array}{c}\text { Triplet } \\
(n=401)\end{array}$ & $D^{b}$ & $\begin{array}{l}\text { Doublet } \\
(n=340)\end{array}$ & $\begin{array}{c}\text { Triplet } \\
(\mathrm{n}=340)\end{array}$ & $D^{b}$ \\
\hline Male sex & $391(68.7 \%)$ & $262(65.3 \%)$ & -7.23 & $232(68.2 \%)$ & $224(65.9 \%)$ & -4.89 \\
\hline Median age (range), y & $66(21-86)$ & $61(22-82)$ & -4.31 & $62(21-83)$ & $62(22-82)$ & -0.63 \\
\hline Mean age $\pm S D, y$ & $64.57 \pm 11.40$ & $58.52 \pm 12.22$ & -4.31 & $61.31 \pm 11.77$ & $60.44 \pm 11.64$ & -0.63 \\
\hline $\mathrm{CEA}, \geq 10 \mathrm{mcg} / \mathrm{L}$ & $177(36.9 \%)$ & $131(37.5 \%)$ & 1.24 & $106(37.3 \%)$ & $118(39.7 \%)$ & 4.99 \\
\hline ECOG PS $\geq 2$ & $121(21.3 \%)$ & $25(6.2 \%)$ & -44.94 & $28(8.2 \%)$ & $25(7.4 \%)$ & -2.98 \\
\hline$\geq 2$ chronic comorbidities & $132(23.2 \%)$ & $51(12.7 \%)$ & -27.61 & $48(14.1 \%)$ & $47(13.8 \%)$ & -0.86 \\
\hline Chronic cardiopathy & $85(14.9 \%)$ & $23(5.7 \%)$ & -30.61 & $21(6.2 \%)$ & $23(6.8 \%)$ & 2.43 \\
\hline $\begin{array}{l}\text { Acute, serious complication } \\
\text { at diagnosis }\end{array}$ & $79(13.9 \%)$ & $43(10.7 \%)$ & -9.75 & $40(11.8 \%)$ & $38(11.2 \%)$ & -1.88 \\
\hline \multicolumn{7}{|l|}{ Primary tumor site } \\
\hline Esophagus & $90(15.8 \%)$ & $61(15.2 \%)$ & -1.65 & $51(15.0 \%)$ & $54(15.9 \%)$ & 2.49 \\
\hline Stomach & $479(84.2 \%)$ & $340(84.8 \%)$ & 1.65 & $289(85.0 \%)$ & $286(84.1 \%)$ & -2.49 \\
\hline \multicolumn{7}{|l|}{ Stage at diagnosis } \\
\hline $\begin{array}{l}\text { Unresectable, locally } \\
\text { advanced }\end{array}$ & $17(3 \%)$ & $28(7 \%)$ & 18.43 & $13(3.8 \%)$ & $14(4.1 \%)$ & 1.54 \\
\hline Metastatic & $552(97 \%)$ & $373(93 \%)$ & -18.43 & $327(96.2 \%)$ & $326(95.9 \%)$ & -1.54 \\
\hline \multicolumn{7}{|l|}{ Surgery of the primary tumor } \\
\hline Resection & $152(26.7 \%)$ & $114(28.4 \%)$ & 3.80 & $96(28.2 \%)$ & $94(27.6 \%)$ & -1.33 \\
\hline Palliative surgery & $37(6.5 \%)$ & $36(9 \%)$ & 9.3 & $24(7.1 \%)$ & $31(9.1 \%)$ & 7.33 \\
\hline No surgery & $380(66.8 \%)$ & $251(62.6 \%)$ & -8.75 & $220(64.7 \%)$ & $215(63.2 \%)$ & -3.12 \\
\hline \multicolumn{7}{|l|}{ Perioperative treatment } \\
\hline $\begin{array}{l}\text { Adjuvant } \\
\text { chemoradiotherapy }\end{array}$ & $28(4.9 \%)$ & $18(4.5 \%)$ & -1.89 & $18(5.3 \%)$ & $14(4.1 \%)$ & -5.67 \\
\hline $\begin{array}{l}\text { Adjuvant } \\
\text { chemotherapy }\end{array}$ & $11(1.9 \%)$ & $5(1.2 \%)$ & -5.66 & $9(2.6 \%)$ & $5(1.5 \%)$ & -7.76 \\
\hline $\begin{array}{l}\text { Perioperative } \\
\text { chemotherapy }\end{array}$ & $22(3.9 \%)$ & $8(2 \%)$ & -11.24 & $16(4.7 \%)$ & $7(2.1 \%)$ & -14.38 \\
\hline $\begin{array}{l}\text { Preoperative } \\
\text { chemotherapy }+ \\
\text { adjuvant radiotherapy }\end{array}$ & $8(1.4 \%)$ & $7(1.7 \%)$ & 2.42 & $7(2.1 \%)$ & $6(1.8 \%)$ & -2.16 \\
\hline Other & $2(0.4 \%)$ & $1(0.2 \%)$ & -3.65 & $2(0.6 \%)$ & $1(0.3 \%)$ & -4.48 \\
\hline \multicolumn{7}{|l|}{ Signet ring cells } \\
\hline No & $337(59.2 \%)$ & $218(54.4 \%)$ & -9.70 & $187(55.0 \%)$ & $194(57.1 \%)$ & 4.23 \\
\hline Yes & $181(31.8 \%)$ & $126(31.4 \%)$ & -0.86 & $112(32.9 \%)$ & $108(31.8 \%)$ & -2.35 \\
\hline Not available & $51(9 \%)$ & $57(14.2 \%)$ & 16 & $41(12.1 \%)$ & $38(11.2 \%)$ & -2.80 \\
\hline
\end{tabular}

Abbreviations: CEA, carcinoembryonic antigen; $\mathrm{D}^{\mathrm{b}}=$ standardized difference; ECOG PS, Eastern Cooperative Group Performance Status scale; FISH, fluorescence in situ hybridization; PSM, propensity score matching.

tantly, the procedure balances out the differences between ECOG PS, chronic comorbidities, stage at diagnosis, presence of extrahepatic metastases, and serious acute cancer-related complication at diagnosis, which were significant covariates for OS in the Cox proportional hazards regression (see following sections). The only covariate that was not balanced after PSM is prior perioperative chemotherapy.

\section{Effect of Triplets Versus Doublets on OS Using the Whole Population}

Patients who received triplets had significantly better OS in a Cox proportional hazards regression 
Triplets or Doublets in Gastric Cancer

\begin{tabular}{|c|c|c|c|c|c|c|}
\hline \multirow[b]{2}{*}{ Characteristics } & \multicolumn{3}{|c|}{ Before PSM } & \multicolumn{3}{|c|}{ After PSM } \\
\hline & $\begin{array}{l}\text { Doublet } \\
(n=569)\end{array}$ & $\begin{array}{c}\text { Triplet } \\
(n=401)\end{array}$ & $D^{b}$ & $\begin{array}{l}\text { Doublet } \\
(n=340)\end{array}$ & $\begin{array}{c}\text { Triplet } \\
(n=340)\end{array}$ & $D^{b}$ \\
\hline \multicolumn{7}{|l|}{ Lauren classification } \\
\hline Intestinal & $259(45.5 \%)$ & $185(46.1 \%)$ & 1.20 & $160(47.1 \%)$ & $156(45.9 \%)$ & -2.40 \\
\hline Diffuse & $197(34.6 \%)$ & $147(36.7 \%)$ & 4.38 & $121(35.6 \%)$ & $125(36.8 \%)$ & 2.49 \\
\hline Mixed & $29(5.1 \%)$ & $21(5.2 \%)$ & 0.45 & $18(5.3 \%)$ & $20(5.9 \%)$ & 2.60 \\
\hline Not available/ unclassifiable & $84(14.8 \%)$ & $48(12 \%)$ & -8.22 & $41(12.1 \%)$ & $39(11.5 \%)$ & -1.85 \\
\hline \multicolumn{7}{|l|}{ Histologic grade } \\
\hline Grade $1-2$ & $221(38.8 \%)$ & $130(32.4 \%)$ & -12.57 & $132(38.8 \%)$ & $116(34.1 \%)$ & -9.79 \\
\hline Grade 3 & $233(40.9 \%)$ & $181(45.1 \%)$ & 8.49 & $136(40.0 \%)$ & $152(44.7 \%)$ & 9.52 \\
\hline Not available & $115(20.2 \%)$ & $90(22.4 \%)$ & 5.37 & $72(21.2 \%)$ & $72(21.2 \%)$ & 0 \\
\hline \multicolumn{7}{|l|}{ HER2 overexpression } \\
\hline $\begin{array}{l}\text { Negative }(0,1+, 2+\text {, and } \\
\text { FISH-) }\end{array}$ & $413(72.6 \%)$ & $281(70.1 \%)$ & -5.53 & $251(73.8 \%)$ & $241(70.9 \%)$ & -6.48 \\
\hline Positive $(3+, 2+$, and $\mathrm{FISH}+)$ & $17(2.9 \%)$ & $13(3.2 \%)$ & 1.74 & $6(1.76 \%)$ & $12(3.52 \%)$ & 10.99 \\
\hline Not available & $139(24.4 \%)$ & $107(26.7 \%)$ & 5.20 & $83(24.4 \%)$ & $876(25.6 \%)$ & 2.77 \\
\hline \multicolumn{7}{|l|}{ Number of metastatic sites } \\
\hline 1 & $198(34.8 \%)$ & $126(31.4 \%)$ & -7.22 & $110(32.4 \%)$ & $108(31.8 \%)$ & -6.48 \\
\hline 2 & $181(31.8 \%)$ & $145(36.2 \%)$ & 9.29 & $112(32.9 \%)$ & $118(34.7 \%)$ & 3.80 \\
\hline 3 & $110(19.3 \%)$ & $77(19.2 \%)$ & -0.25 & $72(21.2 \%)$ & $63(18.5 \%)$ & -6.77 \\
\hline$\geq 4$ & $80(14.1 \%)$ & $53(13.2 \%)$ & -2.62 & $46(13.5 \%)$ & $51(15 \%)$ & 4.29 \\
\hline \multicolumn{7}{|l|}{ Site of metastases } \\
\hline Liver & $220(38.7 \%)$ & $118(29.4 \%)$ & -19.72 & $116(34.1 \%)$ & $110(32.4 \%)$ & -3.60 \\
\hline Peritoneum & $255(44.8 \%)$ & $202(50.4 \%)$ & 11.23 & $160(47.1 \%)$ & $160(48.5 \%)$ & 2.8 \\
\hline Bone & $59(10.4 \%)$ & $39(9.7 \%)$ & -2.32 & $39(11.5 \%)$ & $36(10.6 \%)$ & -2.87 \\
\hline Lung & $69(12.1 \%)$ & $34(8.5 \%)$ & -11.86 & $31(10 \%)$ & $32(9.4 \%)$ & -2.02 \\
\hline \multicolumn{7}{|c|}{ Year of first-line chemotherapy administration } \\
\hline 2008-2009 & $58(10 \%)$ & $61(15.2 \%)$ & 15.7 & $36(10.6 \%)$ & $50(14.7 \%)$ & 12.35 \\
\hline 2010-2011 & $102(17.9 \%)$ & $64(16 \%)$ & -5.06 & $71(20.9 \%)$ & $51(15 \%)$ & -15.41 \\
\hline 2012-2013 & $188(33 \%)$ & $128(31.9 \%)$ & -2.34 & $115(33.8 \%)$ & $107(31.5 \%)$ & -4.90 \\
\hline$\geq 2014$ & $221(38.8 \%)$ & $148(36.9 \%)$ & -3.91 & $118(34.7 \%)$ & $132(38.8 \%)$ & 8.5 \\
\hline
\end{tabular}

Abbreviations: CEA, carcinoembryonic antigen; $\mathrm{D}^{\mathrm{b}}=$ standardized difference; ECOG PS, Eastern Cooperative Group Performance Status scale; FISH, fluorescence in situ hybridization; PSM, propensity score matching.

model compared with patients treated with doublets after adjusting for confounding factors (HR, 0.84; 95\% CI, 0.72-0.98; $P=.035$ ) (Table 2).

\section{Effect of Triplets Versus Doublets on OS, PFS,} Response, and Toxicity Using the PSM-Matched Sample

A significant increase in OS was observed, 11.14 (95\% CI, 9.60-12.68) versus 9.60 months (95\% CI, 8.44-10.75), in patients who receive triplets versus doublets, respectively ( $\mathrm{HR}, 0.77 ; 95 \% \mathrm{CI}$,
0.65-0.92; stratified log-rank test, $P=.004)$. Figure 2 shows Kaplan-Meier curves for OS. After PSM, a difference in PFS is also seen in favor of triplets: 6.97 months (95\% CI, 6.40-7.53) versus 6.11 months (95\% CI, 5.64-6.58; HR, 0.82; 95\% CI, 0.69-0.97; stratified log-rank test, $P=.027$ ). The magnitude of the effect appeared to be similar for anthracycline-based triplets (HR, 0.78; 95\% CI, 0.64-0.94) or docetaxel-based triplets (HR, 0.78; 95\% CI, 0.60-1.009). 
Carmona-Bayonas et al

Table 2. Adverse Events Recorded After PSM

\begin{tabular}{|c|c|c|c|c|}
\hline \multirow[b]{2}{*}{ Toxicity } & \multicolumn{2}{|c|}{ Doublet } & \multicolumn{2}{|c|}{ Triplet } \\
\hline & Total & $\begin{array}{c}\text { Grade } \\
3 / 4\end{array}$ & Total & $\begin{array}{c}\text { Grade } \\
3 / 4\end{array}$ \\
\hline Anemia & $59.6 \%$ & $5.0 \%$ & $68.8 \%$ & $10.7 \%$ \\
\hline Neutropenia & $47.2 \%$ & $18.1 \%$ & $56.5 \%$ & $30.1 \%$ \\
\hline Febrile neutropenia & \multicolumn{2}{|c|}{$6.5 \%$} & \multicolumn{2}{|c|}{$10.4 \%$} \\
\hline Thrombocytopenia & $21.4 \%$ & $1.5 \%$ & $28.0 \%$ & $3.9 \%$ \\
\hline Emesis & $40.1 \%$ & 3.6 & $42.9 \%$ & $4.2 \%$ \\
\hline Diarrhea & $34.1 \%$ & 4.7 & $47.9 \%$ & $6.8 \%$ \\
\hline Stomatitis & $24.6 \%$ & 2.4 & $36.6 \%$ & $4.2 \%$ \\
\hline Fatigue & $66.5 \%$ & 5.0 & $75 \%$ & $8.6 \%$ \\
\hline Hand-Foot syndrome & $26.4 \%$ & 0.9 & $32.4 \%$ & $5.7 \%$ \\
\hline Neuropathy & $49.3 \%$ & 4.5 & $66.4 \%$ & $5.1 \%$ \\
\hline Alopecia & \multicolumn{2}{|c|}{$17.5 \%$} & \multicolumn{2}{|c|}{$69 \%$} \\
\hline $\begin{array}{l}\text { Increased aspartate } \\
\text { aminotransferase }\end{array}$ & $9.2 \%$ & 0.6 & $15.8 \%$ & $1.8 \%$ \\
\hline Hyperbilirubinemia & $6.5 \%$ & 2.1 & $8.6 \%$ & $2.1 \%$ \\
\hline Renal toxicity & $5.0 \%$ & 0.6 & $6.3 \%$ & $0.9 \%$ \\
\hline $\begin{array}{l}\text { Venous } \\
\text { thromboembolic } \\
\text { disease }\end{array}$ & $11.3 \%$ & 4.2 & $11 \%$ & $6 \%$ \\
\hline $\begin{array}{l}\text { Toxicity-related } \\
\text { hospital admission }\end{array}$ & \multicolumn{2}{|c|}{$22.6 \%$} & \multicolumn{2}{|c|}{$30.7 \%$} \\
\hline $\begin{array}{l}\text { Death due to } \\
\text { toxicity }\end{array}$ & \multicolumn{2}{|c|}{$0.6 \%$} & \multicolumn{2}{|c|}{$1.2 \%$} \\
\hline
\end{tabular}

Abbreviation: PSM, propensity score matching.

There were no significant differences in the use of second-lines for doublets and triplets $(47.1 \%$ and $49.6 \%$, respectively; $P=.529)$. Insofar as evaluating the 3-month tumor response with the RECIST 1.1 method was concerned, higher objective response rates were seen with triplets than doublets $(49.6 \%$ vs $39.2 \%$, respectively) in subjects with initial measurable disease, although disease control rates (complete response, partial response, and stable disease) were similar in both groups ( $74.2 \%$ vs $75.2 \%$ ). On the contrary, triplets were seen to be associated with greater overall and grade $3 / 4$ toxicities in comparison with doublets (Table 3), particularly with more grade $3 / 4$ anemia, stomatitis, febrile neutropenia ( $10.4 \%$ vs $6.5 \%)$, hepatic toxicity $(15.8 \%$ vs $9.2 \%)$, and toxicity-related hospitalization $(30.7 \%$ vs $22.6 \%)$. Granulocyte colony-stimulating factor (GCSF) prophylaxis was used in $36.2 \%$ of triplets versus $12 \%$ of doublets $(P<.0001)$. Treatment-related death occurred in 2 triplet-treated patients and 1 doublettreated patient.

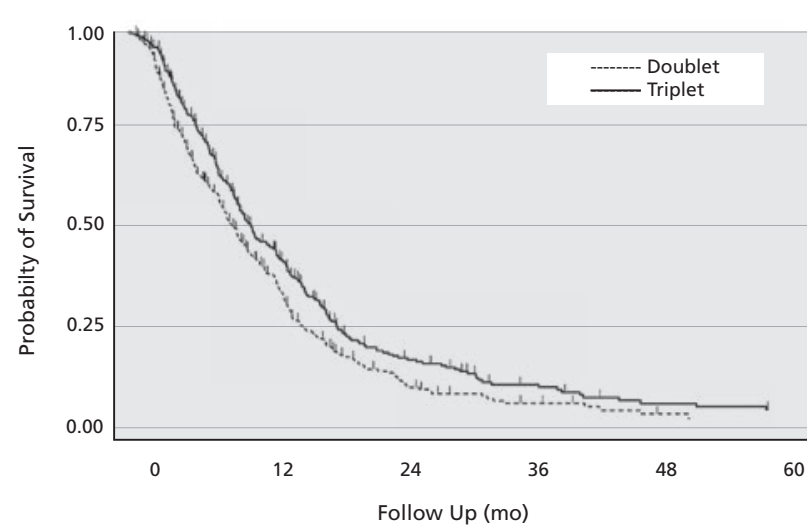

Figure 2. Overall survival, $11.14(95 \% \mathrm{Cl}, 9.60-12.68)$ vs 9.60 months $(95 \% \mathrm{Cl}, 8.44-10.75)$ in patients who received triplets versus doublets, respectively ( $\mathrm{HR}, 0.77 ; 95 \% \mathrm{Cl}, 0.65-0.92$; stratified log-rank test, adjusted for quintiles of the performance status, $P=.004$ ).

\section{Doses Administered in Doublets or Triplets Using the PSM-Matched Sample}

To estimate the possible effect of adding a third drug (anthracycline or docetaxel), oxaliplatin doses were compared in patients who received EOX (epirubicin, oxaliplatin, and capecitabine) versus CAPOX/ FOLFOX (capecitabine and oxaliplatin/folinic acid, 5-fluorouracil, and oxaliplatin), and cisplatin doses in patients treated with XP (capecitabine and cisplatin) versus DCX (docetaxel, cisplatin, and capecitabine). We also evaluated the ability to maintain planned dosing schedules for epirubicin and docetaxel in triplet chemotherapy (Table 4). No clinically significant differences were seen in treatment time, number of cycles administered, accumulated doses, or RDIs for each regimen, with the exception of a reduction in the RDI for cisplatin to less than $85 \%$ with DCX and XP. Nonetheless, the data were not consistent with the hypothesis that anthracycline-based or docetaxelbased triplets are quickly reconverted to doublets in actual clinical practice. To the contrary, the most probable cause for suspending epirubicin or docetaxel was progression and because the complete number of planned cycles was administered, and, less frequently, due to toxicity or patient choice (Table 4).

\section{Treatment Effect After Adjustment With CEM}

With CEM (multivariate L1 distance, 8.353e-16), the effect observed in 602 matched observations was of a similar magnitude, with a weighted HR for OS of the effect (based on CEM weights) of 0.78 (95\% CI, 0.63-0.97; P=.03). 
Triplets or Doublets in Gastric Cancer

\begin{tabular}{|c|c|c|c|c|c|c|c|}
\hline & \multicolumn{3}{|c|}{ Oxaliplatin } & \multicolumn{2}{|c|}{ Cisplatin } & \multirow{2}{*}{$\frac{\text { Epirubicin }}{E O X / E C F}$} & \multirow{2}{*}{$\begin{array}{l}\text { Docetaxel } \\
\text { DCX/DCF/ } \\
\text { DOX/DOF }\end{array}$} \\
\hline & EOX & FOLFOX6 & CAPOX & XP & DCX/DCF & & \\
\hline Median number of cycles (range) & $6(1-12)$ & $8(-16)$ & $5(-11)$ & $6(-15)$ & $6(-19)$ & $6(-12)$ & $5(-19)$ \\
\hline Median of treatment duration (wk) & 18 & 19 & 16 & 19 & 19 & 18 & 20 \\
\hline Mean cumulative dose $\left(\mathrm{mg} / \mathrm{m}^{2}\right)$ & 674 & 685 & 690 & 420 & 345 & 247 & 338 \\
\hline Mean dose/cycle $\left(\mathrm{mg} / \mathrm{m}^{2} /\right.$ cycle $)$ & 122 & 81 & 122 & 71 & 60 & 48 & 64 \\
\hline Mean dose intensity (mg/m²/wk) & 37 & 37 & 38 & 22 & 18 & 15 & 19 \\
\hline Mean, dose density & $86 \%$ & $87 \%$ & $88 \%$ & $76 \%$ & $82 \%$ & $88 \%$ & $87 \%$ \\
\hline \multicolumn{8}{|l|}{ Reason for withdrawal } \\
\hline Toxicity & $16 \%$ & $23 \%$ & $25 \%$ & $12 \%$ & $12 \%$ & $18 \%$ & $13 \%$ \\
\hline Progression & $36 \%$ & $25 \%$ & $47 \%$ & $52 \%$ & $33 \%$ & $33 \%$ & $35 \%$ \\
\hline Planned treatment completed & $31 \%$ & $29 \%$ & $14 \%$ & $30 \%$ & $40 \%$ & $35 \%$ & $39 \%$ \\
\hline Patient refusal & $2 \%$ & $5 \%$ & $2 \%$ & $1 \%$ & $1 \%$ & $2 \%$ & $2 \%$ \\
\hline Other & $7 \%$ & $11 \%$ & $7 \%$ & $4 \%$ & $5 \%$ & $7 \%$ & $6 \%$ \\
\hline Not available & $8 \%$ & $7 \%$ & $5 \%$ & $1 \%$ & $9 \%$ & $5 \%$ & $5 \%$ \\
\hline
\end{tabular}

Abbreviations: CAPOX, capecitabine, oxaliplatin; DCF, docetaxel, cisplatin, fluorouracil; DCX, docetaxel, cisplatin, capecitabine; DOF, docetaxel, oxaliplatin, fluorouracil; DOX, docetaxel, oxaliplatin, capecitabine; ECF, epirubicin, cisplatin, fluorouracil; EOX, epirubicin, oxaliplatin, capecitabine; FOLFOX6, fluorouracil, oxaliplatin; PSM, propensity score matching; XP, capecitabine, cisplatin.

\section{Discussion}

According to a recent meta-analysis, the use of chemotherapy triplets for AGC has proven to be superior to doublet therapy, although the gain in benefit is modest compared with the increment in toxicity. ${ }^{15}$ This conclusion raises doubts as to the applicability of the results to conditions of real clinical practice, given that advanced age, deterioration in general status, and chronic comorbidities are usual..$^{23}$

In our community practice registry, after generating a sample having a balanced distribution of baseline variables with PSM, the survival analysis factored for the use of triplets or doublets confirms these findings, with a difference in median OS of more than 1.8 months in favor of the former (HR, $0.77 ; P=.004)$. Nevertheless, this is achieved at the expense of increasing toxicities, including hospitalizations due to toxicity in patients who receive triplets compared to those receiving doublets. All this could also entail a pharmacoeconomic impact that would increase support treatment costs (eg, G-CSF), particularly in more toxic regimens.

The main difference with the aforementioned meta-analysis ${ }^{15}$ might be that treatment with docetaxel-containing triplets had less weight in our series. In our registry, doublets were used more than triplets, at a proportion of 3:2. However, $70 \%$ of the triplets in our series are based on the addition of epirubicin, whereas docetaxel-based regimens accounted for the remaining $30 \%$.

In a previous meta-analysis by Wagner et al, ${ }^{24}$ when anthracycline-based triplets were compared with CF-like regimens, a significant improvement in OS was suggested (HR, $0.77 ; 95 \% \mathrm{CI}, 0.62-0.95$ ), although these results were based on 3 small heterogeneous trials with comparators that were scantly commensurable to the current ones. This paucity of data from RCTs with an appropriate sample size is one of the justifications for performing analyses such the present one. Another rationale is to confirm possible preconceptions about the use of triplets in everyday clinical practice, especially in the context of vulnerable patients.

These results do not bear out the hypothesis that the scant incremental benefit of triplets is due to them being quickly reconverted into doublets as a consequence of possible early toxicities. Rather, what is seen is that, despite the greater toxicity of triplets compared with doublets, this toxicity is rarely cause for discontinuation of anthracycline or docetaxel and the doses administered exceed 85\% of those planned in both cases. Likewise, the addition 


\begin{tabular}{|c|c|c|c|c|}
\hline Covariate & b & HR & $95 \% \mathrm{Cl}$ & $P$ Value \\
\hline Sex, female & 0.1488 & 1.1605 & $0.9926-1.3568$ & .0633 \\
\hline Age, y & -0.005654 & 0.9944 & $0.9876-1.0012$ & .1069 \\
\hline ECOG PS $\geq 2$ & 0.6966 & 2.0069 & $1.6436-2.4505$ & $<.0001$ \\
\hline$\geq 2$ chronic comorbidities & 0.2278 & 1.2558 & $1.0437-1.5111$ & .0164 \\
\hline Acute, serious complication at diagnosis & 0.3322 & 1.3941 & $1.1226-1.7312$ & .0028 \\
\hline \multicolumn{5}{|l|}{ Primary tumor site } \\
\hline Stomach vs esophagus & -0.2866 & 0.7508 & $0.6098-0.9245$ & .0072 \\
\hline No primary tumor surgery & 0.2581 & 1.2945 & $1.1712-1.4308$ & $<.0001$ \\
\hline \multicolumn{5}{|l|}{ Previous treatments } \\
\hline Adjuvant chemoradiotherapy & 0.5790 & 1.7842 & $1.2476-2.5516$ & .0016 \\
\hline Adjuvant chemotherapy & 1.0096 & 2.7445 & $1.4301-5.2670$ & .0025 \\
\hline Perioperative chemotherapy & 0.6776 & 1.9691 & $1.2985-2.9859$ & .0015 \\
\hline \multicolumn{5}{|l|}{ Lauren classification } \\
\hline Diffuse vs intestinal & 0.1561 & 1.1689 & $0.9745-1.4021$ & .0943 \\
\hline Unknown & 0.2066 & 1.2295 & $0.9752-1.5501$ & .0821 \\
\hline \multicolumn{5}{|l|}{ HER2 status } \\
\hline$\geq 3$ vs others & -0.5490 & 0.5775 & $0.3361-0.9926$ & .0481 \\
\hline Unknown & 0.1852 & 1.2034 & $1.0158-1.4257$ & .0331 \\
\hline \multicolumn{5}{|l|}{ Histologic grade } \\
\hline Grade 2 vs grade 1 & 0.3913 & 1.4789 & $1.0965-1.9948$ & .0108 \\
\hline Grade 3 vs grade 1 & 0.2815 & 1.3251 & $0.9777-1.7960$ & .0710 \\
\hline Not available & 0.3612 & 1.4350 & $1.0353-19891$ & .0310 \\
\hline \multicolumn{5}{|l|}{ Site of metastases } \\
\hline Liver & 0.2449 & 1.2775 & $1.0839-1.5057$ & .0037 \\
\hline Peritoneum & 0.2329 & 1.2623 & $1.0715-1.4870$ & .0056 \\
\hline Bone & 0.5670 & 1.7630 & $1.4050-2.2121$ & $<.0001$ \\
\hline \multicolumn{5}{|l|}{ First-line chemotherapy } \\
\hline Triplet vs doublet & -0.1670 & 0.8462 & 0.7248 to 0.9880 & 0.0355 \\
\hline
\end{tabular}

Abbreviations: ECOG PS, Eastern Cooperative Group Performance Status scale; HR, hazard ratio.

of these drugs does not appear to impact the RDI of platins and fluoropyrimidines in triplets, in relation to the RDI of these same drugs when they are part of a doublet regimen, except in the case of cisplatin in combination with oral capecitabine.

This study has several limitations. First, when carefully performed, PSM is capable of balancing out an important part of the asymmetries in baseline covariates, resulting from systematic treatment selection in nonrandomized series. Nonetheless, when projecting many covariates on a scalar propensity score, there is a risk of generating additional imbalances. ${ }^{25}$ Moreover, in our registry, PSM has appar- ently been effective in mitigating the imbalance of most of the known covariates, with the exception of the percentage of perioperative treatment, which is a variable affecting both prognosis and treatment selection. Still, the survival analysis is similar, whether the $12 \%$ of patients with prior perioperative treatment are excluded or not. Additionally, CEM data processing was applied including this variable and it basically confirmed the same results. As a second limitation, the concept of "doublet" or "triplet" encompasses several different types of regimens. Insofar as it is doubtful that all of the regimens are exactly the same in terms of safety or efficacy, the analysis 
we report has the purview of a general panoramic to be confirmed in future research. Third, most of the data are from before the value of second-line anti-VEGFR2 (ramucirumab) was formally established. This can be a source of added uncertainty, for instance, if the treatment sequence offset the comparative ineffectiveness of first-line doublets.

Finally, the data analyses we present must be considered retrospective, with the accuracy limitations inherent in this type of study. Nonetheless, the main end points (PFS and OS) are solid outcomes and most of the variables (eg, chemotherapy schedules, pathology), are reliably recorded in medical histories. In contrast, despite the matching techniques used, performance status might continue to behave as a residual confounding factor. This is suggested by the fact that it is the main determinant in the choice of doublets versus triplets, while simultaneously influencing OS more than the treatment effect (Table 4 and eTable 3 ).

With all the preceding considerations, this analysis is surely of interest as a hypothesis generator. First, therapy intensification through the use of triplets appears to be a suitable choice in patients with good PS and no chronic comorbidities, and, particularly, in those with unresectable locally advanced tumors. This may also be the case for patients who have not received prior perioperative treatment. Second, although oncologists also seem to choose treatment intensification for patients with more aggressive cancers (eg, odds ratio of 2.8 for poorly differentiated tumors; eTable 3 ), our analysis did not provide evidence suggesting that these patients would obtain greater benefit from this practice.

\section{Conclusions}

The data in this community practice-based, multicenter registry reveal that triplet therapies are feasible; they are not rapidly reconverted to doublets in real conditions, nor do they appear to ostensibly affect the other components of the treatment regimen compared with the administration of these same drugs as a doublet. The survival benefit would be discrete, albeit significant. In exchange, triplets are more toxic, especially regarding hematologic toxicity and mucositis, leading to more hospital admissions due to adverse events. Finally, the existence of patient groups that would benefit differently from each treatment modality remains to be ascertained.

\section{Acknowledgments}

The authors wish to thank Priscilla Chase Duran for editing the manuscript, and Natalia Cateriano, Miguel Vaquero, and IRICOM S.A. for the support of the Web site registry.

\section{References}

1. Siegel RL, Miller KD, Jemal A. Cancer statistics, 2016. CA Cancer J Clin 2016;66:7-30

2. Bang YJ, Van Cutsem E, Feyereislova A, et al. Trastuzumab in combination with chemotherapy versus chemotherapy alone for treatment of HER2. positive advanced gastric or gastro-oesophageal junction cancer (ToGA): a phase 3, open-label, randomised controlled trial. Lancet 2010;376:687697.

3. Tokunaga A, Onda M, Okuda T, et al. Clinical significance of epidermal growth factor (EGF), EGF receptor, and c-erbb-2 in human gastric cancer. Cancer 1995;75(6 Suppl):1418-1425.

4. Ishida T, Tsujisaki M, Hanzawa Y, et al. Significance of erbB-2 gene product as a target molecule for cancer therapy. Scand J Immunol 1994;39:459-466.

5. Garrido M, Fonseca PJ, Vieitez JM, et al. Challenges in first line chemotherapy and targeted therapy in advanced gastric cancer. Expert Rev Anticancer Ther 2014;14:887-900.

6. Van Cutsem E, Moiseyenko VM, Tjulandin S, et al. Phase III study of docetaxel and cisplatin plus fluorouracil compared with cisplatin and fluorouracil as first-line therapy for advanced gastric cancer: a report of the V325 Study Group. J Clin Oncol 2006;24:4991-4997.

7. Ochenduszko S, Puskulluoglu M, Konopka K, et al. Comparison of efficacy and safety of first-line palliative chemotherapy with EOX and mDCF regimens in patients with locally advanced inoperable or metastatic HER2-negative gastric or gastroesophageal junction adenocarcinoma: a randomized phase 3 trial. Med Oncol 2015;32:1-8.

8. Wang J, Xu R, Li J, et al. Randomized multicenter phase III study of a modified docetaxel and cisplatin plus fluorouracil regimen compared with cisplatin and fluorouracil as first-line therapy for advanced or locally recurrent gastric cancer. Gastric Cancer 2016;19:234-244.

9. Van Cutsem E, Boni C, Tabernero J, et al. Docetaxel plus oxaliplatin with or without fluorouracil or capecitabine in metastatic or locally recurrent gastric cancer: a randomized phase II study. Ann Oncol 2015;26:149-156.

10. Rivera F, Massutí B, Salcedo M, et al. Phase II trial of miniDOX (reduced dose docetaxel-oxaliplatin-capecitabine) in "suboptimal" patients with advanced gastric cancer (AGC). TTD 08-02. Cancer Chemother Pharmacol 2015;75:319-324.

11. Wagner A, Grothe W, Haerting J, et al. Chemotherapy in advanced gastric cancer: a systematic review and meta-analysis based on aggregate data. J Clin Oncol 2006;24:2903-2909.

12. Ross $\mathrm{P}$, Nicolson M, Cunningham D, et al. Prospective randomized trial comparing mitomycin, cisplatin, and protracted venous-infusion fluorouracil (PVI 5-FU) with epirubicin, cisplatin, and PVI 5-FU in advanced esophagogastric cancer. J Clin Oncol 2002;20:1996-2004.

13. A randomized, comparative study of combination chemotherapies in advanced gastric cancer: 5-fluorouracil and cisplatin (FP) versus 5-fluorouracil, cisplatin, and 4'-epirubicin (FPEPIR). Kyoto Research Group for Chemotherapy of Gastric Cancer (KRGCGC). Anticancer Res 1992;12(6B):1983-1988.

14. Kim TW, Choi SJ, Ahn JH, et al. A prospective randomized phase III trial of 5-fluorouracil and cisplatin (FP) versus epirubicin, cisplatin, and 5-fu $(E C F)$ in the treatment of patients with previously untreated advanced gastric cancer (AGC). Eur J Cancer 2001;37:S314.

15. Mohammad NH, Ter Veer E, Ngai L, et al. Optimal first-line chemotherapeutic treatment in patients with locally advanced or metastatic esophagogastric carcinoma: triplet versus doublet chemotherapy: a systematic literature review and meta-analysis. Cancer Metastasis Rev 2015;34:429-441.

16. Austin PC. A critical appraisal of propensity-score matching in the medical literature between 1996 and 2003. Stat Med 2008;27:2037-2049.

17. Austin PC. A tutorial and case study in propensity score analysis: an application to estimating the effect of in-hospital smoking cessation counseling on mortality. Multivariate Behav Res 2011;46:119-151. 
18. Austin PC. Balance diagnostics for comparing the distribution of baseline covariates between treatment groups in propensity-score matched samples. Stat Med 2009;28:3083-3107.

19. Iacus SM, King G, Porro G. Multivariate matching methods that are monotonic imbalance bounding. J Amer Stat Assoc 2011;106:345-361.

20. Iacus SM, King G, Porro G. Causal Inference Without Balance Checking: Coarsened Exact Matching. Available at: https:/gking.harvard.edu/ files/gking/files/political_analysis-2011-iacus-pan_mpr013.pdf. Accessed October 4, 2016.

21. Leuven E, Sianesi B. PSMATCH2: Stata Module to Perform Full Mahalanobis and Propensity Score Matching, Common Support Graphing, and Covariate Imbalance Testing. Available at: https://ideas.repec.org/c/ boc/bocode/s432001.html. Accessed October 4, 2016.

22. Blackwell M, Iacus $S$, King G, Porro G. cem: Coarsened exact matching in Stata. Stata J 2009;9:524-546.

23. Koppert LB, Janssen-Heijnen ML, Louwman MW, et al. Comparison of comorbidity prevalence in oesophageal and gastric carcinoma patients: a population-based study. Eur J Gastroenterol Hepatol 2004;16:681-688.

24. Wagner AD, Unverzagt $S$, Grothe $W$, et al. Chemotherapy for advanced gastric cancer. Cochrane Database Syst Rev 2010:CD004064.

25. King G, Nielsen R. Working Paper. Why Propensity Scores Should Not Be Used for Matching. Available at: http://j.mp/1sexgVw. Accessed October 4, 2016

See JNCCN.org for supplemental online content. 